\title{
Ansiedad Dental en Adultos Chilenos que Concurren a un Servicio de Salud Primaria
}

\author{
Dental Anxiety in Chilean Adults Who Attend a Primary Care Health Service
}

\author{
Matías Ríos-Erazo; Andrea Herrera-Ronda*; Pilar Barahona-Salazar*; Yerko Molina-Muñoz*; \\ Patricia Cadenasso-Salinas $^{* *}$; Verónica Zambrano-Canelo ${ }^{* *}$ \& Gonzalo Rojas-Alcayaga*
}

RÍOS-ERAZO, M.; HERRERA-RONDA, A.; BARAHONA-SALAZAR, P.; MOLINA-MUÑOZ, Y.; CADENASSO-SALINAS, P.; ZAMBRANO-CANELO, V. \& ROJAS-ALCAYAGA, G. Ansiedad dental en adultos chilenos que concurren a un servicio de salud primaria. Int. J. Odontostomat., 10(2):261-266, 2016.

RESUMEN: El objetivo fue identificar el nivel de ansiedad dental en una muestra de adultos chilenos que concurren a un servicio de atención de salud primaria. Se realizó un estudio transversal, obteniendo una muestra de 174 adultos, con edades entre los 20 y 70 años, pertenecientes al área urbana de Santiago de Chile, y que acudían en calidad de acompañantes de niños(as) que asistían a atención dental. Se registraron datos socio-demográficos y fecha de último control dental. Se aplicó la escala de ansiedad dental de Corah. Se realizaron estadísticos descriptivos, prueba t, prueba U de Mann Whitney y prueba de Spearman. Se trabajó con un error de significancia del $5 \%$. Un 37,9\% de la muestra presentó ansiedad dental, en sus niveles moderado (16,1\%), severo $(13,2 \%)$ y fobia dental $(8,6 \%)$. A nivel educacional, los sujetos con educación básica completa presentaron la mayor frecuencia $(57,1 \%)$ de ansiedad, mientras que los de educación superior incompleta presentaron la menor frecuencia (25\%). No se observaron asociaciones entre ansiedad dental y edad, sexo, nivel educacional, índice per cápita y último control dental. La muestra de adultos encuestados de Santiago de Chile presentó altos niveles de ansiedad dental en comparación con países desarrollados. Se recomienda realizar más estudios en dicha población para determinar las variables que explican el problema de la ansiedad dental en la población chilena.

PALABRAS CLAVE: ansiedad dental, adultos, ciencias del comportamiento, salud primaria, estudio transversal.

\section{INTRODUCCIÓN}

La salud oral depende, en gran medida, de la adquisición de ciertas conductas y la evitación de otras, lo que finalmente se traduce en un estilo de vida. La conducta de asistir periódicamente al dentista, requiere de una valoración positiva de ésta, junto con eliminar barreras que interfieren en la adherencia a tratamiento, como la ansiedad dental. Ésta es definida como una respuesta multisistémica ante una creencia de peligro o amenaza relacionada con la atención dental, la cual es una experiencia individual, subjetiva, y que varía entre las personas (Al-Namankany et al., 2012).

La ansiedad dental es un constructo multidimensional que posee componentes cognitivos, fisiológicos y motores (Ríos Erazo et al., 2014). Con- siderada como uno de los principales factores que conducen a la evitación de la atención odontológica (Al-Namankany et al.) y a la disminución de la salud oral del individuo (Armfield, 2010), distintos estudios dan cuenta de su asociación con un aumento en el número de dientes cariados y de enfermedades gingivales (Armfield et al., 2009; Eitner et al., 2006; Rantavuori et al., 2014). Por otra parte, la ansiedad dental también es considerada un eficaz predictor de problemas de manejo conductual del paciente en el sillón dental (Gustafsson et al., 2010).

Diversos estudios muestran que las frecuencias de ansiedad dental en países europeos varían entre el 4,2 \% y el 29,9\% (Eitner et al.; Enkling et al., 2006;

Área de Ciencias del Comportamiento, Instituto de Investigación en Ciencias Odontológicas, Facultad de Odontología, Universidad de Chile, Santiago, Chile.

* Centro de Salud Familiar Dr. Alfonso Leng, Municipalidad de Providencia, Santiago, Chile.

*** Centro de Salud Familiar Recreo, Municipalidad de San Miguel, Santiago, Chile.

Fuente de Financiamiento: Fondo Nacional en Salud (FONIS) 
RíoS-ERAZO, M.; HERRERA-RONDA, A.; BARAHONA-SALAZAR, P.; MOLINA-MUÑOZ, Y.; CADENASSO-SALINAS, P.; ZAMBRANO-CANELO, V. \& ROJAS-ALCAYAGA, G. Ansiedad dental en adultos chilenos que concurren a un servicio de salud primaria. Int. J. Odontostomat., 10(2):261-266, 2016.

Grimshaw et al., 2012; Hakeberg et al., 1992; Kirova et al., 2010; Moore et al., 1993; Nicolas et al., 2007; Pohjola et al., 2008). En Oceanía y Estados Unidos, la prevalencia de ansiedad dental oscila entre los 12,5 $\%$ y 16,1 \% (Armfield et al., 2006; Locker et al., 2001; Thomson et al., 1996), mientras que en Asia las tasas van del $30 \%$ al 50,2 \% (Marya et al., 2012; Schwarz \& Birn, 1995). A nivel latinoamericano, solo en Brasil existen estudios que reportan datos de ansiedad dental y cuyas frecuencias están cercanas al $20 \%$ (de Carvalho et al., 2012; do Nascimento et al., 2011). Sumado a las limitadas investigaciones existentes, debe considerarse que el estudio de la ansiedad dental en la población resulta complejo, ya que muchos de los pacientes que presentan este problema no se atienden en el centro de salud correspondiente debido al temor que les genera, mostrando un importante ausentismo al tener horas agendadas con el dentista (Enkling et al.; Erten et al., 2006). Al no existir datos en población chilena, y considerando el ausentismo que presenta la población clínica con ansiedad dental, se hace necesario estudiar este problema en población nacional, por lo que el objetivo de este trabajo es identificar el nivel de ansiedad dental en una muestra de adultos chilenos que concurren en calidad de acompañantes a un servicio de atención de salud primaria de la Región Metropolitana.

\section{MATERIAL Y MÉTODO}

Este estudio contó con la aprobación del comité de ética de la Facultad de Odontología de la Universidad de Chile. Se realizó un estudio transversal, a través de un muestreo intencionado, que incluyó a la población de adultos que acudían en calidad de acompañantes de escolares de 6 años, que asistían a control odontológico en el Centro de Salud Familiar Recreo, de la comuna de San Miguel, del Centro de Salud Familiar Dr. Alfonso Leng, de la Comuna de Providencia, y del Centro de Salud Bellavista, de la comuna de la Florida. Se les invitó a participar del estudio y en caso de acceder se procedió a la firma del consentimiento informado.

Inicialmente se les aplicó una encuesta que contenía información sociodemográfica como sexo, edad, nivel educacional, ingreso per cápita y último control dental. Para evaluar el nivel de ansiedad dental del sujeto, se utilizó el Dental Anxiety Scale (DAS), creado por Norman Corah, el cual consta de cuatro preguntas, con cinco alternativas de respuesta, con puntuaciones de 1 a 5 , y con categorizaciones que van desde relajado (4 a 7 puntos), inquieto ( 8 a 11 puntos), ansiedad moderada (12 a 14 puntos), ansiedad severa (15 a 17 puntos) y fobia dental (18 a 20 puntos) (Corah, 1969). Este escala fue seleccionada por ser considerada la más utilizada en la medición de la ansiedad dental en población adulta (Gordon et al., 2013).

Para el análisis de datos, se utilizaron estadísticos descriptivos con el objetivo de caracterizar la muestra en las variables de interés, por medio de tablas de frecuencias para las variables ordinales y nominales, y promedio y desviación estándar para las variables escalares.

Para evaluar la diferencia en la ansiedad dental según edad, se aplicó la prueba t de student para muestras independientes, mientras que para ver las diferencias según nivel educacional, y última visita al dentista, se aplicó la prueba de U-Mann Whitney. Para evaluar la relación entre ansiedad dental e ingreso per cápita, última visita al dentista y edad, se realizó un análisis de correlación de Spearman. Se consideró una confiabilidad del $95 \%$.

\section{RESULTADOS}

En total fueron 174 los adultos participantes del estudio (89,1\% mujeres y $10,9 \%$ hombres), con edades que fluctuaban entre los 20 y 70 años (Media: 37,4; DE: 10,96).

Con respecto al nivel educacional, un 42,3\% de la muestra poseía educación media completa, y solo un 3,6 \% básica incompleta. En relación a la última visita al dentista, un $62,6 \%$ de los sujetos evaluados manifestaron que al menos hace un año que no visitaban al dentista. Dentro de ese porcentaje, un 20,9 $\%$ no había asistido a sus controles con el odontólogo hace más de 5 años.

En relación al nivel de ansiedad dental que presenta la muestra, un $37,9 \%$ presenta ansiedad dental, en sus niveles moderado, severo y fobia dental (Tabla I).

Al observar la presencia de ansiedad dental según el nivel educacional de la muestra evaluada, las personas de educación básica completa presentan porcentualmente un mayor nivel de ansiedad dental que los demás grupos. Según grupo etario, los su- 
jetos entre 60 a 70 años presentan porcentualmente un mayor nivel de ansiedad dental que los demás grupos etarios (Tabla II).

Finalmente, al chequear las asociaciones entre la ansiedad dental y la edad, índice per cápita y última visita al dentista, no se observan asociaciones significativas. Lo mismo ocurre al chequear si existen diferencias significativas entre la presencia/ausencia de ansiedad dental según nivel educacional, edad y última visita al dentista (Tabla III).

Tabla I. Características Socio-Demográficas, controles dentales y ansiedad dental de la muestra en estudio.

\begin{tabular}{llc}
\hline Variable & & $\mathbf{n}(\%)$ \\
\hline Sexo & Hombre & $19(10,9)$ \\
& Mujer & $155(89,1)$ \\
& Básica Incompleta & $6(3,6)$ \\
& Básica Completa & $7(4,2)$ \\
& Media incompleta & $27(16,1)$ \\
& Media Completa & $71(42,3)$ \\
& Superior Incompleta & $21(12,5)$ \\
& Superior Completa & $36(21,4)$ \\
Última visita al dentista & $0-1$ años & $61(37,4)$ \\
& 1-2 años & $37(22,7)$ \\
& 2-3 años & $18(11)$ \\
& 3-4 años & $7(4,3)$ \\
& 4-5 años & $6(3,7)$ \\
& Más de 5 años & $34(20,9)$ \\
Nivel de Ansiedad & Relajado & $54(31)$ \\
& Inquieto & $54(31)$ \\
& Ansiedad Moderada & 28 \\
& Ansiedad Severa & 23 \\
& Fobia Dental & $15(8,6)$ \\
\hline
\end{tabular}

Tabla II. Ansiedad dental, nivel educacional y grupo etario.

\begin{tabular}{llcc}
\hline \multirow{2}{*}{ Variable } & & \multicolumn{2}{c}{ Ansiedad dental } \\
\cline { 3 - 4 } Nivel Educacional & Básica Incompleta & 33,3 & Ausencia \% \\
& Básica Completa & 57,1 & 66,7 \\
& Media incompleta & 48,1 & 42,9 \\
& Media Completa & 38,0 & 51,9 \\
& Superior Incompleta & 42,9 & 62,0 \\
Grupo Etario & Superior Completa & 25,0 & 57,1 \\
& 20 a 29 años & 33,3 & 75,0 \\
& 30 a 39 años & 36,6 & 66,7 \\
& 40 a 49 años & 37,1 & 63,4 \\
& 50 a 59 años & 55,6 & 62,9 \\
& 60 a 70 años & 62,1 & 44,4 \\
\end{tabular}

Tabla III. Tabla de correlaciones y diferencias de la ansiedad dental con variables de estudio.

\begin{tabular}{llcc}
\hline & & Estadístico & $\boldsymbol{p}$ \\
\hline Asociación con ansiedad & Edad & Rho: 0,122 & 0,141 \\
dental & Ingreso per cápita & Rho: $-0,105$ & 0,194 \\
& Ultima consulta dental & Rho: 0,131 & 0,310 \\
Diferencias en presencia/ & Edad & t: $-1,142$ & 0,255 \\
ausencia de ansiedad & Nivel educacional & U: 2822,5 & 0,833 \\
& Ultima consulta al dentista & U: 3101,5 & 0,917 \\
\hline
\end{tabular}


RíOS-ERAZO, M.; HERRERA-RONDA, A.; BARAHONA-SALAZAR, P.; MOLINA-MUÑOZ, Y.; CADENASSO-SALINAS, P.; ZAMBRANO-CANELO, V. \& ROJAS-ALCAYAGA, G. Ansiedad dental en adultos chilenos que concurren a un servicio de salud primaria. Int. J. Odontostomat., 10(2):261-266, 2016.

\section{DISCUSIÓN}

A partir de los resultados obtenidos de la muestra estudiada, es posible indicar que la presencia de ansiedad dental en adultos chilenos $(37,9 \%)$ es mayor a la de países desarrollados como Alemania (13,2 \%) (Enkling et al.), Turquía (21,3 \%) (Firat et al., 2006), Francia (13,5\%) (Nicolas et al.), Australia (16,1\%) (Armfield et al., 2006) o Nueva Zelanda (12,5\%) (Locker et al.). Al realizar una comparación con países subdesarrollados, la presencia de ansiedad dental es similar a la de Bulgaria (35,5 \%) (Kirova et al.). En relación a la fobia dental obtenida en la muestra $(8,6 \%)$, los resultados son similares a estudios realizados en Brasil, donde un 9,5\% de los adultos estudiados presentaba fobia dental (do Nascimento et al.), y son menores a los presentados en Bulgaria $(11,7 \%)$ (Kirova et al.) y Alemania (11\%) (Enkling et al.).

En relación a las diferencias por sexo en torno a la presencia de ansiedad dental, en el presente estudio no se vieron diferencias, al contrario de los hallazgos de muchos estudios que si dan cuenta que las mujeres presentan mayor ansiedad dental que los hombres (Armfield et al., 2006; de Carvalho et al.; do Nascimento et al.; Enkling et al.; Firat et al.; Hakeberg et al.; Marya et al.; Stabholz \& Peretz, 1999). Sin embargo, la importante diferencia muestral entre hombres $(10,9 \%)$ y mujeres $(89,1 \%)$ impiden obtener resultados concluyentes.

Con respecto al grupo etario, no se identificaron diferencias por grupo. Sin embargo, se observa una tendencia en los adultos entre 60 a 70 años los cuales presentan mayores niveles de ansiedad dental que otros rangos de edad, lo que coincide con los hallazgos en Australia (40 a 64 años) (Armfield et al., 2006), pero no con los estudios en India (20 a 30 años) (Marya et al.), Israel (35 a 49 años) (Stabholz \& Peretz) o Alemania (19 a 29 años) (Eitner et al.), lo que da cuenta de la importante heterogeneidad en estos resultados. Bajo este contexto, sería recomendable aumentar el tamaño muestral para chequear si estas tendencias muestran diferencias estadísticas.

Un dato interesante es que frente a la pregunta de cuándo fue la última vez que visitó al odontólogo, un $20,9 \%$ indicó que llevaba 5 años o más sin visitar al dentista. Estos datos son similares a los reportados en la última Encuesta Nacional de Salud realizada en Chile el año 2010 (MINSAL, 2010), donde un 27,1 \% de los sujetos entre 45 a 64 años, indicaron que hace más de 5 años que no visitaban al dentista, y un 4,5\% señaló no haberlo visitado nunca.

Una de las limitaciones de esta investigación resulta ser la no equitativa distribución por sexo, lo que impidió la realización de ciertos análisis como la comparación por género. En un próximo estudio sería recomendable aumentar el tamaño muestral para solucionar dicho problema lo que además permitiría evaluar las diferencias que podrían existir según grupo etario.

\section{AGRADECIMIENTOS}

Este trabajo fue financiado por el Fondo Nacional en Salud (FONIS), dependiente de CONICYT, a través del Proyecto FONIS SA11I2025, titulado: "Impacto en la adherencia a tratamiento de una intervención cognitivo-conductual para disminuir ansiedad dental en niños y niñas de 6 años beneficiarios del programa de salud oral integral en servicios de atención primaria de la Región Metropolitana."

RÍOS-ERAZO, M.; HERRERA-RONDA, A.; BARAHONASALAZAR, P.; MOLINA-MUÑOZ, Y.; CADENASSO-SALINAS, P.; ZAMBRANO-CANELO, V. \& ROJAS-ALCAYAGA, G. Dental anxiety in Chilean adults who attend a primary care health service. Int. J. Odontostomat., 10(2):261-266, 2016.

ABSTRACT: The objective of this study is to identify the level of dental anxiety in a sample of Chilean adults who attend a service of primary health care. A cross-sectional study was carried out. The sample consisted of 174 caregivers of pediatric dental patients, aged between 20 and 70 years, belonging to the urban area of Santiago de Chile. A questionnaire that included socio-demographic information (sex, age, educational level, income per capita index and last dental visit) were registered. Corah's Dental Anxiety Scale was applied. Descriptive statistics and t-test, U-Mann Whitney test and Spearman test was used. An error of significance of $5 \%$ was applied. $37.9 \%$ of the sample shows dental anxiety in their moderate levels $(16.1 \%)$, severe $(13.2 \%)$ and dental phobia (8.6\%). Of the subjects who had dental anxiety, the majority had moderate anxiety (42.4\%). In an educational level, subjects with complete basic education have the highest rate $(57.1 \%)$ of anxiety, while incomplete higher education had the lowest rate $(25 \%)$. Adults between 50 and 59 years reported greater presence of dental anxiety. There were not significant associations between dental anxiety and age, sex, educational level, per capita rate and last dental visit. The sample of adults surveyed in Santiago de Chile presented high levels of dental anxiety compared to other developed 
countries. We recommend further studies in this population to determine the variables that explain the problem of dental anxiety in the Chilean population.

KEY WORDS: dental anxiety, adults, behavioral sciences, dental fear, primary health care, cross sectional study.

\section{REFERENCIAS BIBLIOGRÁFICAS}

Al-Namankany, A.; de Souza, M. \& Ashley, P. Evidencebased dentistry: analysis of dental anxiety scales for children. Br. Dent. J., 212(5):219-22, 2012.

Armfield, J. M. How do we measure dental fear and what are we measuring anyway? Oral Health Prev. Dent., 8(2):107-15, 2010.

Armfield, J. M.; Slade, G. D. \& Spencer, A. J. Dental fear and adult oral health in Australia. Community Dent. Oral Epidemiol., 37(3):220-30, 2009.

Armfield, J. M.; Spencer, A. J. \& Stewart, J. F. Dental fear in Australia: who's afraid of the dentist? Aust. Dent. J., 51(1):78-85, 2006.

Corah, N. L. Development of a dental anxiety scale. J. Dent. Res., 48(4):596, 1969.

de Carvalho, R. W.; Falcão, P. G.; Campos, G. J.; Bastos, A. S.; Pereira, J. C.; Pereira, M. A.; Cardoso, M. S. \& Vasconcelos, B. C. Anxiety regarding dental treatment: prevalence and predictors among Brazilians. Cien. Saude Colet., 17(7):1915-22, 2012.

do Nascimento, D. L.; da Silva Araújo, A. C.; Gusmão, E. S. \& Cimões, R. Anxiety and fear of dental treatment among users of public health services. Oral Health Prev. Dent., 9(4):329-37, 2011.

Eitner, S.; Wichmann, M.; Paulsen, A. \& Holst, S. Dental anxiety--an epidemiological study on its clinical correlation and effects on oral health. J. Oral Rehabil., 33(8):588-93, 2006.

Enkling, N.; Marwinski, G. \& Jöhren, P. Dental anxiety in a representative sample of residents of a large German city. Clin. Oral Investig., 10(1):84-91, 2006.

Erten, H.; Akarslan, Z. Z. \& Bodrumlu, E. Dental fear and anxiety levels of patients attending a dental clinic. Quintessence Int., 37(4):304-10, 2006.

Firat, D.; Tunc, E. P. \& Sar, V. Dental anxiety among adults in Turkey. J. Contemp. Dent. Pract., 7(3):75-82, 2006.
Gordon, D.; Heimberg, R. G.; Tellez, M. \& Ismail, A. I. A critical review of approaches to the treatment of dental anxiety in adults. J. Anxiety Disord., 27(4):365-78, 2013.

Grimshaw, G. P.; Boyle, C. A. \& Newton, T. Dental anxiety levels in British servicemen and women. Community Dent. Health, 29(3):239-42, 2012.

Gustafsson, A.; Broberg, A.; Bodin, L.; Berggren, U. \& Arnrup, K. Dental behaviour management problems: the role of child personal characteristics. Int. J. Paediatr. Dent., 20(4):242-53, 2010.

Hakeberg, M.; Berggren, U. \& Carlsson, S. G. Prevalence of dental anxiety in an adult population in a major urban area in Sweden. Community Dent. Oral Epidemiol., 20(2):97-101, 1992.

Kirova, D. G.; Atanasov, D. T.; Lalabonova, C. K. \& Janevska, S. Dental anxiety in adults in Bulgaria. Folia Med. (Plovdiv), 52(2):49-56, 2010.

Locker, D.; Poulton, R. \& Thomson, W. M. Psychological disorders and dental anxiety in a young adult population. Community Dent. Oral Epidemiol., 29(6):456-63, 2001.

Marya, C. M.; Grover, S.; Jnaneshwar, A. \& Pruthi, N. Dental anxiety among patients visiting a dental institute in Faridabad, India. West Indian Med. J., 61(2):187-90, 2012.

Ministerio de Salud (MINSAL). Encuesta Nacional de Salud ENS Chile 2009-2010. Santiago de Chile, Ministerio de Salud, 2015. Disponible en: http:// w e b. mins al. cl/ portal/url/it e m / bcb03d7bc28b64dfe040010165012d23.pdf

Moore, R.; Birn, H.; Kirkegaard, E.; Brødsgaard, I. \& Scheutz, F. Prevalence and characteristics of dental anxiety in Danish adults. Community Dent. Oral Epidemiol., 21(5):292-6, 1993.

Nicolas, E.; Collado, V.; Faulks, D.; Bullier, B. \& Hennequin, M. A national cross-sectional survey of dental anxiety in the French adult population. BMC Oral Health, 7:12, 2007.

Pohjola, V.; Lahti, S.; Tolvanen, M. \& Hausen, H. Dental fear and oral health habits among adults in Finland. Acta Odontol. Scand., 66(3):148-53, 2008.

Rantavuori, K.; Sihvonen, A.; Tolvanen, M. \& Lahti, S. The significance of previous invasive and orthodontic treatment experiences on children's dental fear. Eur. J. Orthod., 36(4):409-13, 2014.

Ríos Erazo, M.; Herrera Ronda, A. \& Rojas Alcayaga, G. Ansiedad dental: Evaluación y tratamiento. Av. Odontoestomatol., 30(1):39-46, 2014. 
RÍOS-ERAZO, M.; HERRERA-RONDA, A.; BARAHONA-SALAZAR, P.; MOLINA-MUÑOZ, Y.; CADENASSO-SALINAS, P.; ZAMBRANO-CANELO, V. \& ROJAS-ALCAYAGA, G. Ansiedad dental en adultos chilenos que concurren a un servicio de salud primaria. Int. J. Odontostomat., 10(2):261-266, 2016.

Schwarz, E. \& Birn, H. Dental anxiety in Danish and Chinese adults--a cross-cultural perspective. Soc. Sci. Med., 41(1):123-30, 1995.

Stabholz, A. \& Peretz, B. Dental anxiety among patients prior to different dental treatments. Int. Dent. J., 49(2):90-4, 1999.

Thomson, W. M.; Stewart, J. F.; Carter, K. D. \& Spencer, A. J. Dental anxiety among Australians. Int. Dent. J., 46(4):320-4, 1996.
Dirección para Correspondencia:

Prof. Matias Rios Erazo

Área Ciencias del Comportamiento

Facultad de Odontología

Universidad de Chile

Santiago

CHILE

Email: matiasrios@gmail.com

Recibido : 15-10-2016

Aceptado: 01-06-2016 